\title{
AAN Approach for Monitoring the Arcing Discharges by using Image Processing Techniques
}

\author{
R. Mahalakshmi \\ Associate Professor, Saveetha Engineering College, \\ Chennai-602105, India \\ B.Indhira Priyadharsini \\ Student , ME. EST, Saveetha Engineering College, \\ Chennai-602105, India
}

\begin{abstract}
Insulator flashover under pollution is one of the most important problems for power transmission. This paper is dedicated to monitor discharges activity through arcing discharges pattern recognition using a combination of efficient image processing and classification algorithms. Images are extracted from recorded videos of flashover process over a plane model insulator under various contamination levels. Then an algorithm is designed and tested from the image that we have obtained from the recorded video. The algorithm is mainly classified into four stages. First, Otsu image segmentation algorithm is initially applied on images. By means of which we obtain the segmented image with noises. In order to eliminate unwanted noises such as light reflections on the insulator model we go for morphological filtering by combining erosion and dilation operations is computed. Third step deals with labeling the connected components and obtaining the four parameters namely $\mathrm{Nl}, \mathrm{Np}, \mathrm{L}$ and $\mathrm{W}$ respectively. These parameters are given as input for the fourth step. Finally the fourth stage deals with image classification in which three classifier algorithms(KNN,SVM,Navie Bayes) are being used and the arcing discharge is being monitored which helps in knowing about the proper functioning of the insulator.
\end{abstract}

Keywords - KNN, SVM, Flash over, insulator pollution and arcing discharges.

\section{INTRODUCTION}

Nowadays, due to the combination of growing demand for electricity and the need to upgrade or replace existing equipment of the electrical network, massive investments will be required to meet future needs. Such investments are meaningless if efforts are not consented to assure security of such equipment. Indeed, the first challenge consists in transmitting electricity (covering the distances between producer and consumer). During this transmission process, insulators play a primary role by maintaining electrical insulation ranging from distribution to transmission lines and supporting mechanical load between a conductor and the ground. However, transmission and distribution systems remain vulnerable to many faults. One of the most harming is the electrical flashover of insulators under pollution. In order to minimize risks of flashover occurrence on high voltage insulators and assure safety of equipment, operators and users, the flashover phenomenon has to be thoroughly studied. The major cause of this phenomenon is the accumulation of pollution on the insulator surface. Many research reported that pollution layer distribution is generally non-uniform.[1-8]

M. A. Douar et al [4-7] analyzed the flashover process on a plane insulating surface under non-uniform pollution and examined the frequency characteristics of the leakage current (LC). The measurements of phase angle between LC and applied voltage indicate that the equivalent impedance of the insulator could be simulated by ResistorCapacitor (RC) circuit with a high capacitive effect engendered by the reestablished clean band. This effect decreases with the appearance of electric discharges. Using Standard Deviation Multi Resolution Analysis (STDMRA) representation of LC, authors reported good correlation between the insulator surface state and details of LC obtained through Discrete Wavelet Transform (DWT) decomposition. Recently, B. Moula et al [8] used this same technique to detect the eventual presence of partial arcs activity over non-uniformly polluted glass surface. A group 
of authors introduces Acoustic Emission (AE) as a novel discharges monitoring method on insulators under contamination conditions. Contamination caused insulator flashovers produce frequent outages in severely contaminated areas. Lines closer to the ocean are in more danger of becoming contaminated. Several countermeasures have been proposed to improve insulator performance.

The most frequently used methods are:

$\begin{array}{ll}\checkmark & \text { Increasing leakage distance } \\ \checkmark & \text { Semiconducting glaze } \\ \checkmark & \text { Periodic washing } \\ \checkmark & \text { Periodic cleaning } \\ \checkmark & \text { Replacement } \\ \checkmark & \text { Covering with silicon rubber } \\ \checkmark & \text { Covering with petroleum. }\end{array}$

\section{1 INCREASINGLEAKAGEDISTANCE}

1) Increasing leakage distance by increasing the number of units or by using fog-type insulators. The disadvantages of the larger number of insulators are that both the polluted and the impulse flashover voltages increase. The latter jeopardizes the effectiveness of insulation coordination because of the increased strike distance, which increases the overvoltage at substations.

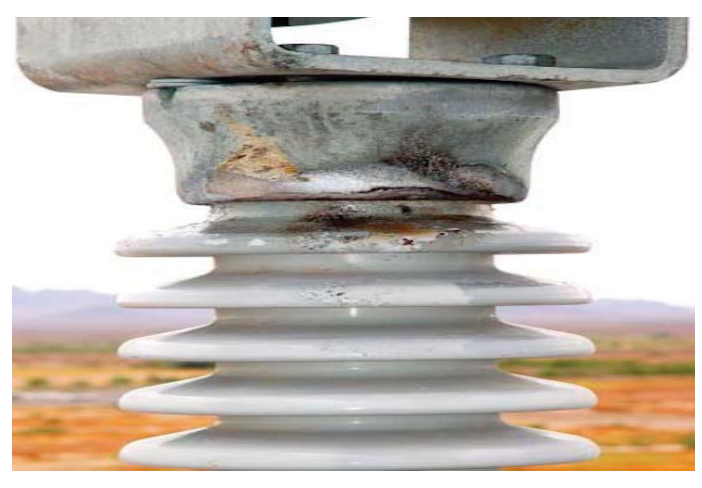

\section{I.2. SEMICONDUCTING GLAZE}

FIG.1: Flashed $230 \mathrm{KV}$ Porcelain Insulator

Application insulators are covered with a semiconducting glaze. A constant leakage current flows through the semiconducting glaze. This current heats the insulator's surface and reduces the moisture of the pollution. In addition, the resistive glaze provides an alternative path when dry bands are formed. The glaze shunts the dry bands and reduces or eliminates surface arcing. The resistive glaze is exceptionally effective near the ocean.

\section{I.3. PERIODICWASHING}

Periodic washing of the insulators with high- pressure water. The transmission lines are washed by a large truck carrying water and pumping equipment Trained personnel wash the insulators by aiming the water spray toward the strings. Substations are equipped with permanent washing systems. High-pressure nozzles are attached to the towers and water is supplied from a central pumping station. Safe washing requires spraying large amounts of 
water at the insulators in a short period of time. Fast washing prevents the formation of dry bands and pollutioncaused flashover. However, major drawbacks of this method include high installation and operational costs.

\section{I.4. PERIODIC CLEANING}

Periodic cleaning of the insulators by high pressure driven abrasive material. Periodic cleaning by materials such as ground corn cobs or walnut shells. This method provides effective cleaning, but cleaning of the residual from the ground is expensive and environmentally undesirable.

\section{I.5. REPLACEMENT}

Replacement of porcelain insulators with non-ceramic insulators. On-ceramic insulators have better pollution performance, which eliminates short-term pollution problems at most sites.However; insulator aging may affect the long-term performance.

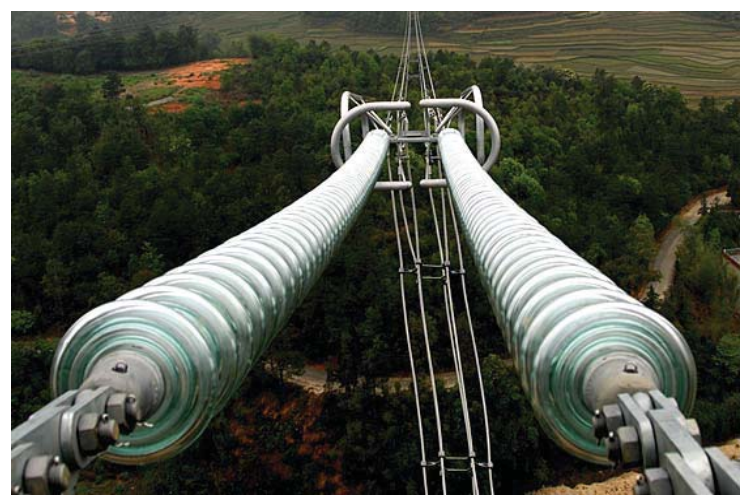

FIG.2: Glass Insulator String For High Voltage Overhead Transmission Line

\section{METHODOLOGY\&IMPLEMENTATION}

In our project the occurrence of flashover is being recorded and then the images are extracted from the recorded video, then image processing algorithms are being implemented which is of four stages. In the first stage OTSU Image segmentation, Secondly Morphological filtering, third stage deals with feature extraction and finally image classification.

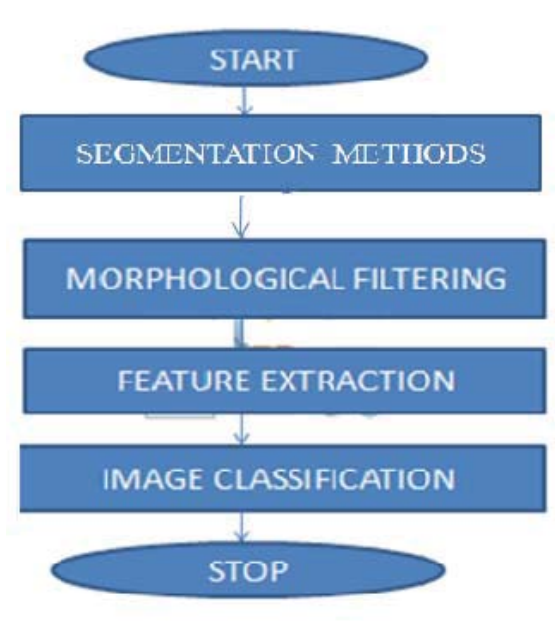

FIG.3. Flow Chart of The Proposed System

A. IMAGE SEGMENTATION: 
Image segmentation is a process by which each pixel of an image is being labeled according to the characteristics which is being shared. Let us consider an image $R$, which is being separated into number of regions $R 1, R 2 \ldots R n$, by means of image segmentation and it is governed with set of rules:

A) $\mathrm{Ri}$ is a connected set $\mathrm{i}=1,2 \ldots \mathrm{n}$.

B) $\mathrm{Ri} \cap \mathrm{Rj}=\varnothing$ for all $\mathrm{i}$ and $\mathrm{j}, \mathrm{i} \neq \mathrm{j}$

C) $Q$ (Ri) $=$ True for $i=1,2$, .n.

D) Q ( Ri U Rj) =False for ad joint regions, Ri and Rj. Where Q (Ri) is a logical predicate.

The rules described above mentions about continuity, one to one relationship, homogeneity and non-repeatability of the pixels after segmentation respectively.

OTSU Image segmentation:

Otsu method which performs clustering based image thresholding, or the conversion of gray level image to binary image .The algorithm considers that the image contains two classes of pixels (foreground pixels and background pixels), then it will be calculating the optimum threshold for the two classes so their intra class variance is minimal and inter class variance is maximum.

Algorithm:

1. The histogram and probabilities of each intensity level to be computed.

2. Set up initial $\omega_{\mathrm{i}(0)}$ and $\mu_{i}(0)$

3. Step through all possible thresholds $t=1 \ldots \ldots$ maximum intensity value update $\omega_{i \text { and }} \mu_{i}$ and compute $\sigma_{b}^{2}(\mathrm{t})$

4. Desired threshold corresponds to the maximum $\sigma_{b}^{2}(t)$ Notations:

$\omega_{\mathrm{i}}$ - Probabilities of two classes separated by a threshold $\mathrm{t}$

$\mu_{i}$ - Class mean., $\sigma_{f}^{*}(\mathrm{t})$ - Class variance.

\section{A. MORPHOLOGICAL FILTERING:}

Set theory is the language of Mathematical Morphology. An object in an image which is represented by the sets in Mathematical Morphology. In binary images, the elements in the sets are members of the 2D integer space Z2. Gray scale images are sets whose components are in Z3.In this case two components of each element of the set refers to the coordinates of a pixel, and the third component corresponds to its intensity value. Some basic operations in Set theory are,

Fundamental operators of Morphological processing are Erosion and Dilation.

(1) Erosion:

With $\mathrm{A}$ and $\mathrm{B}$ as sets in $\mathrm{Z} 2$, the erosion of $\mathrm{A}$ by $\mathrm{B}$, denoted $\mathrm{A} \odot \mathrm{B}$ is defined as

$$
\mathrm{A} \ominus \mathrm{B}=\left\{\mathrm{z} \mid(\mathrm{B})_{\mathrm{z}} \subseteq \mathrm{A}\right\}
$$

(2) Dilation:

With $A$ and $B$ as sets in $Z^{2}$ the dilation of $a$ by $B$ denoted $A \oplus B$ is defined as $\mathrm{A} \oplus \bar{B}=\left\{\mathrm{Z} \mid(B)_{\mathrm{z}} \mathrm{n} A=\emptyset\right\}-------(2)$

\section{B. FEATURE EXTRACTION:}

Before extracting the features, various image processing techniques which includes binarization, thresholding, resizing, normalization etc. is being applied on the sampled image. Then feature extraction techniques are applied. The features that are mainly considered for this includes number of the connected labeled components $\left(\mathrm{N}_{\mathrm{l}}\right)$ the number of pixels, the length and the width of the largest connected component region ( $\mathrm{N}_{\mathrm{p}}, \mathrm{L}$ and $\mathrm{W}$ respectively).By labeling the connected components the features are obtained. 


\section{IMAGE CLASSIFICATION}

KNN Classification:

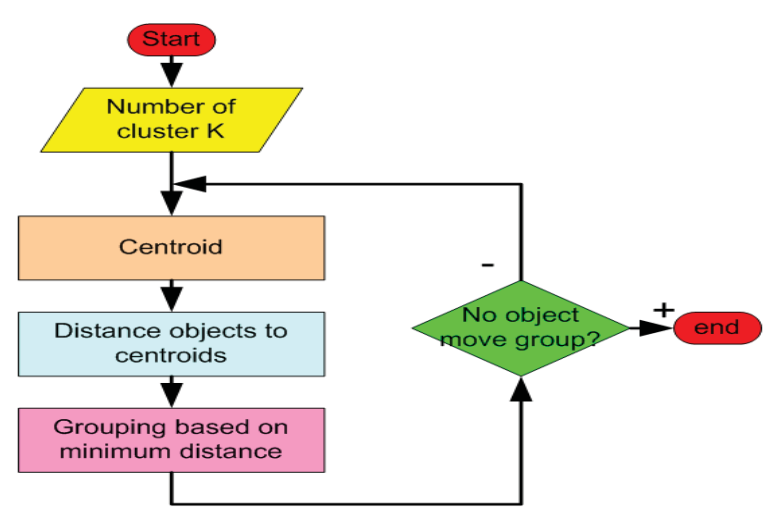

FIG.4: Flow Chart of KNN

In pattern recognition K-Nearest Neighbor is a non-parametric method used for classification. In both the cases, the input consists of the $\mathrm{K}$ closest training examples in the feature space. The output depends on whether KNN is used for classification or regression. The step wise procedure is represented in the above flowchart.

\section{RESULTS AND DISCUSSIONS}

The images were tested in Mat lab and the Simulations are shown. An MRI image obtained from the drive data base is fed as input. Input image is a RGB image.

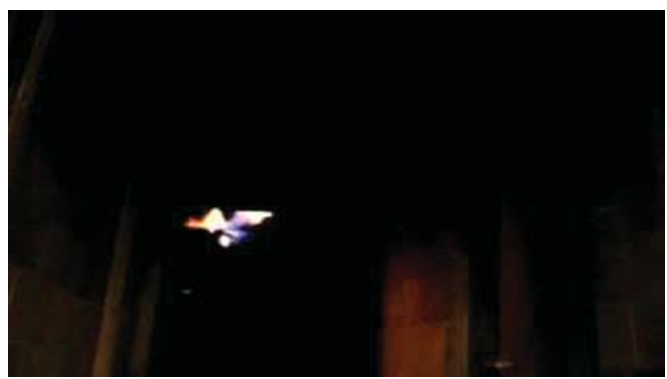

FIG.5: Input Image

Segmented input image which is converted from RGB to gray scale image.

Segmentation Image

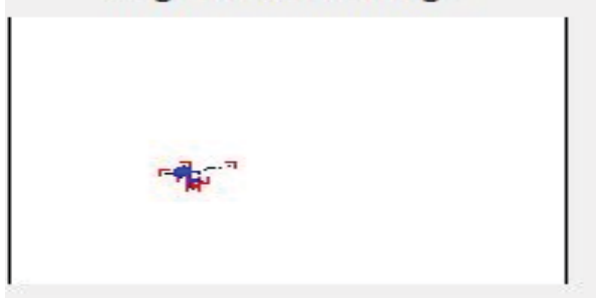

FIG.6: Segmented Image 


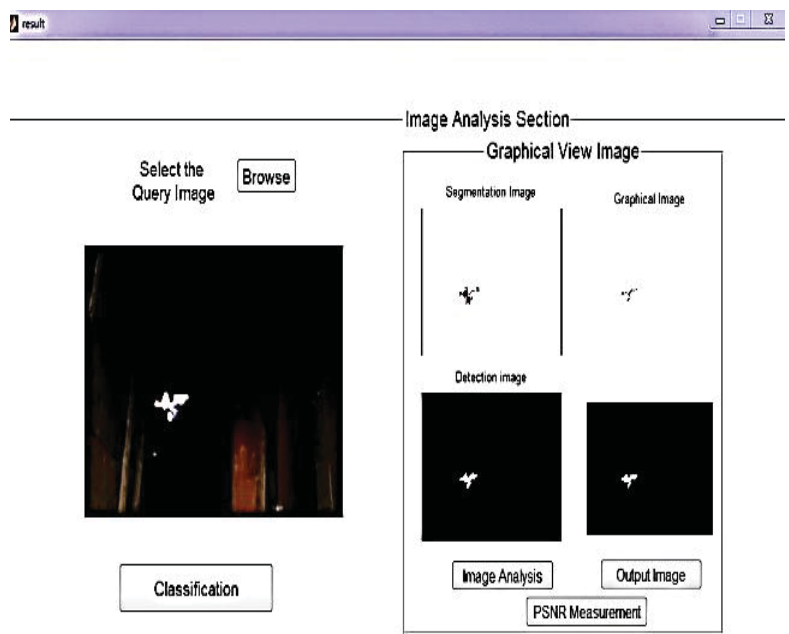

FIG.7: Output Image

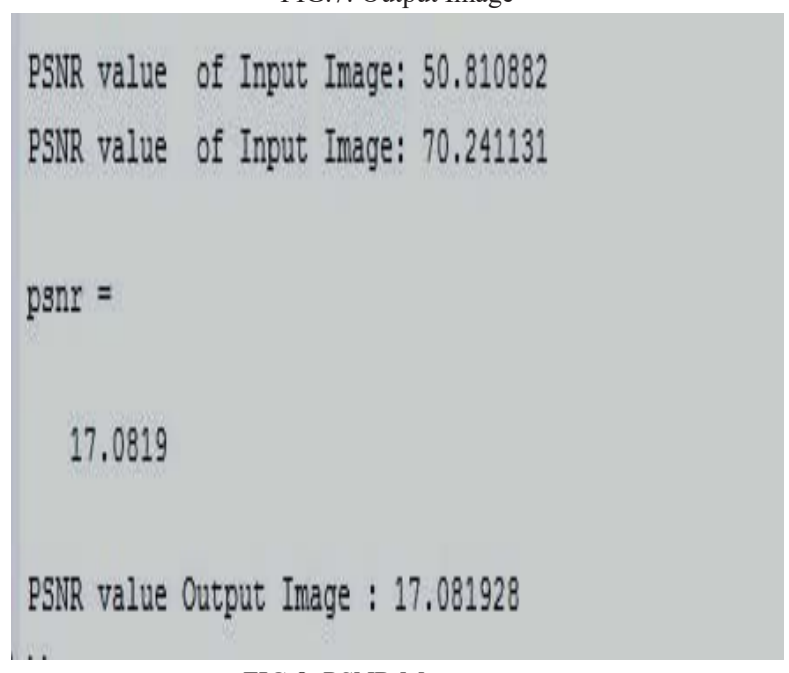

FIG.8: PSNR Measurement

\section{CONCLUSION}

The work described in this paper has introduced a novel methodology to analyze discharges activity and monitor insulation flashover. Experiments were established on a plane insulating surface for better visibility of appearing discharges. Through a proposed algorithm, four indicators are selected to characterize discharges activity and to discern between arcing and non-arcing discharges on the insulating surface. Further, pattern classification (Knn, Naïve Bayes and SVM) is processed to automate arcing discharges recognition. In this work, only KNN classification has been used and Naïve Bayes and SVM can be used for further enhancement.

\section{REFERENCES}

[1] M.A. El Koshairy and F.A.M. Rizk, "Comportement des Isolateurs des Lignes de Transport de Très Haute Tension Dans les Conditions Désertiques”, CIGRE, Rapport 33-05, Paris, France, 1970.

[2] M. Teguar, A. Mekhaldi and A. Boubakeur, "Algorithm for HV Insulators Flashover under Discontinuous pollution", Archives of Electr. Eng., Vol. LI, No.2, pp 137-146, 2002.

[3] M.Teguar, A.Mekhaldi, A.Bouhafs and A.Boubakeur, "Comportement des Surfaces Isolantes sous Pollution Non-Uniforme", Buletinul Institutului Politehnic Din Iași by Universitatea Tehnică "Gheorghe Asachi", Tomul XLVIII (LII), Iași, Romania, pp. 239-244, 2002.

[4] M. A. Douar, A. Mekhaldi and M. C. Bouzidi, "Flashover Process and Frequency Analysis of the Leakage Current on Insulator Model under non-Uniform Pollution Conditions", IEEE Trans. Dielectrics and Electrical Insul., Vol. 17, No. 4, pp. 1284 -1297, 2010. 
[5] M. A. Douar, A. Mekhaldi and M. C. Bouzidi, "Frequency analysis of the leakage current under non uniform polluted conditions on one insulator plane model”, IEEE Conf. Electr. Insul. Dielectr. Phenomena (CEIDP), pp. 1-4, 2010.

[6] M. A. Douar, A. Mekhaldi and M. C. Bouzidi, "Time-scale analysis of the applied voltage waveform in uniform pollution conditions as a new monitoring technique of the insulator flashover", IEEE 10th Int'l. Conf. Solid Dielectrics (ICSD), pp. 1-4, 2010.

[7] M. A. Douar, A. Mekhaldi and M. C. Bouzidi, "Welch periodogram analysis of the leakage current on insulator model under wetted contaminated conditions for flashover prediction", IEEE Mediterranean Electrotechnical Conf. (MELECON), pp. 1636-1641, 2010.

[8] B. Moula, A. Mekhaldi, M.Teguar and A. Haddad, "Characterization of Discharges on non-Uniformly Polluted Glass Surfaces Using a Wavelet Transform Approach”, IEEE Trans. Dielectr. Electr. Insul. Vol. 20, No.4, pp. 1457-1466, 2013. 